University of Nebraska - Lincoln

DigitalCommons@University of Nebraska - Lincoln

Mechanical \& Materials Engineering Faculty

Publications

Mechanical \& Materials Engineering,

Department of

2012

\title{
Damage progression from impact in layered glass modeled with peridynamics
}

\author{
Florin Bobaru \\ University of Nebraska-Lincoln, fbobaru2@unl.edu \\ Youn Doh $\mathrm{Ha}$ \\ Kunsan National University, Korea \\ Wenke Hu \\ University of Nebraska-Lincoln, huwenke_nbu@hotmail.com
}

Follow this and additional works at: https://digitalcommons.unl.edu/mechengfacpub

Bobaru, Florin; Ha, Youn Doh; and Hu, Wenke, "Damage progression from impact in layered glass modeled with peridynamics" (2012). Mechanical \& Materials Engineering Faculty Publications. 99.

https://digitalcommons.unl.edu/mechengfacpub/99

This Article is brought to you for free and open access by the Mechanical \& Materials Engineering, Department of at DigitalCommons@University of Nebraska - Lincoln. It has been accepted for inclusion in Mechanical \& Materials Engineering Faculty Publications by an authorized administrator of DigitalCommons@University of Nebraska Lincoln. 


\title{
Damage progression from impact in layered glass modeled with peridynamics
}

\author{
Florin Bobaru, ${ }^{1}$ Youn Doh $\mathrm{Ha}^{2}$ and Wenke $\mathrm{Hu}^{1}$
}

1. Department of Mechanical and Materials Engineering, University of Nebraska-Lincoln, Lincoln, Nebraska, 68588-0526 USA; email fbobaru2@unl.edu

2. Department of Naval Architecture, Kunsan National University, and National Creative Research Initiatives Center for Isogeometric Optimal Design, Korea

\begin{abstract}
Dynamic fracture in brittle materials has been difficult to model and predict. Interfaces, such as those present in multilayered glass systems, further complicate this problem. In this paper we use a simplified peridynamic model of a multilayer glass system to simulate damage evolution under impact with a high-velocity projectile. The simulation results are compared with results from recently published experiments. Many of the damage morphologies reported in the experiments are captured by the peridynamic results. Some finer details seen in experiments and not replicated by the computational model due to limitations in available computational resources that limited the spatial resolution of the model, and to the simple contact conditions between the layers instead of the polyurethane bonding used in the experiments. The peridynamic model uncovers a fascinating time-evolution of damage and the dynamic interaction between the stress waves, propagating cracks, interfaces, and bending deformations, in three-dimensions.
\end{abstract}

Keywords: Peridynamics, Dynamic fracture, Impact, Brittle fracture, Glass

\section{Introduction}

Glass laminates have become important in recent years and several experimental studies have been dedicated to the behavior of these structures under dynamic loading ([1], [2], [3]). Experiments for high-velocity impact on multilayered glass structures demonstrate very complex damage patterns with significant differences between the types of fracture and characteristics of damage in each layer. While some common features are observed, especially for layers 3 to 6 in the seven-layer laminate investigated in [1], the first layer, the second, and the last layer exhibit distinct damage morphologies. In itself a complex material, glass undergoes a variety of damage patterns under impact, including: crack branching (bifurcation of a crack), crack-path instability and crack curving, successive branching events, circumferential or ripple cracking, micro-cracking, etc. The work published in [1] is a recent attempt of describing in detail the impact damage from a high-velocity projectile onto a glass laminate with the goal of serving as a guide and a calibration for numerical models for penetration resistance of glass. The present contribution attempts to numerically model a simplified version of the physical system used in [1], but at the 
same physical scale. For this we use the nonlocal continuum model called peridynamics ([4]). While we employ the simplest peridynamic constitutive model for a generic brittle material and the boundary conditions imposed in the model are slightly different from the conditions in the experiments, the results show that, at least to first order, the peridynamic model obtains most of the damage morphology observed in the experiments. Future enhancements for more accurate representation of the boundary conditions as well modeling the effect of presence of binding polyurethane thin layers between the glass sheets (not included in the simplified model discussed here), could lead to quantitative predictive simulations of penetration resistance of multi-layer glass laminates using Peridynamics.

\section{Brief review of the peridynamic formulation}

The peridynamic formulation of continuum mechanics assumes that material points separated by a finite distance interact directly with each other ([4]). Such an interaction is called nonlocal interaction. The purpose for this formulation is to remove difficulties that classical continuum models face when dealing with fracture, or more generally, with discontinuous fields that have to be differentiated. We note that the peridynamic nonlocal interaction does not necessarily mean or refer to a "direct" physical interaction (like the one between atoms or small scale structures), but rather it is to be considered an "effective interaction distance" or an "effective lengthscale" of a continuum model in a dynamic problem (see [5]). An in-depth discussion of the connections and differences between peridynamics and other nonlocal models is provided in [6]. The peridynamic formulation [4] uses integration of nodal forces instead of spatial derivatives in the equations of motion and thus it does not face the mathematical inconsistencies that the classical formulation faces at material discontinuities. The peridynamic equations of motion at a point $\mathbf{x}$ and time $t$ are:

$$
\rho \ddot{\mathbf{u}}(\mathbf{x} ; t)=\int_{H_{\mathbf{x}}} \mathbf{f}\left(\mathbf{u}\left(\mathbf{x}^{\prime}, t\right)-\mathbf{u}(\mathbf{x}, t), \mathbf{x}^{\prime}-\mathbf{x}\right) \mathrm{d} \mathbf{x}^{\prime}+\mathbf{b}(\mathbf{x}, t)
$$

where $\ddot{\mathbf{u}}$ is the acceleration vector field, $\mathbf{u}$ is the displacement vector field, $\mathbf{b}$ is a prescribed body force intensity, and $\rho$ is mass density. Also, $\mathbf{f}$ is the pairwise force function in the peridynamic bond that connects material points $\mathbf{x}$ and $\mathbf{x}^{\prime}$. The internal subregion $H_{\mathbf{x}}$ (see Figure 1 ) is defined as

$$
H_{\mathbf{x}}=\left\{\mathbf{x}^{\prime} \in \Re_{0}:\left|\mathbf{x}-\mathbf{x}^{\prime}\right|<\delta\right\}
$$

where $\delta$ is the horizon, the "size" of the nonlocal interaction. A discussion about the meaning, selection, and use

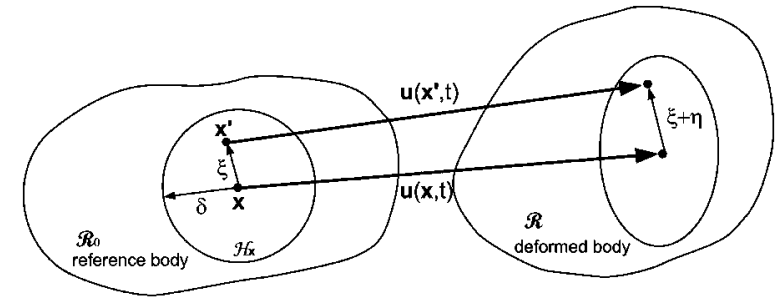

Figure 1. The deformation of a peridynamic bond.

of the peridynamic horizon is given in [5]. Note that no spatial derivatives appear in Equation (1).

A micro-elastic material [4] is defined when the pairwise force derives from a micro-elastic potential $\omega$ :

$$
\mathbf{f}(\eta, \xi)=\frac{\partial \omega(\eta, \xi)}{\partial \eta}
$$

where $\xi=\mathbf{x}^{\prime}-\mathbf{x}$ is the relative position and $\eta=\mathbf{u}\left(\mathbf{x}^{\prime}, t\right)-$ $\mathbf{u}(\mathbf{x}, t)$ is the relative displacement (see Figure 1) between points $\mathbf{x}$ and $\mathbf{x}^{\prime}$. A linear micro-elastic material (the force magnitude depends linearly on the relative elongation magnitude) is obtained if we take

$$
\omega(\eta, \xi)=\frac{c(\xi) s^{2}\|\xi\|}{2}
$$

where $c(\xi)$ is called the micromodulus function and

$$
s=\frac{\|\eta+\xi\|-\|\xi\|}{\|\xi\|}
$$

is the relative elongation of a bond. The corresponding pairwise force is derived from Equations (3-4):

$$
f(\eta, \xi)= \begin{cases}\frac{\xi+\eta}{\|\xi+\eta\|} c s, & \|\xi\| \leq \delta \\ 0, & \|\xi\|>\delta\end{cases}
$$

The micromodulus function is required to satisfy certain conditions of regularity (see [7]), however, the set of allowable functions is quite large. For homogeneous deformations (when $s$ is constant over the entire domain), the relations between the elastic material properties and the micromoduli are given in, for example, [8] for 3D, [9] for 2D, and [10] for 1D. Here we use the "constant" micromodulus in 3D. The elastic strain energy density $W(\mathbf{x})$ at a point $\mathbf{x}$ is obtained by integrating the micro-elastic potential (Equation 4) over the horizon region:

$$
\begin{aligned}
W(\mathbf{x})= & \frac{1}{2} \int_{H \mathbf{x}} \omega(\eta, \xi) \mathrm{d} \mathbf{x}^{\prime}=\frac{1}{2} \int_{0}^{\delta} \frac{C S^{2} r}{2} 4 \pi r^{2} \mathrm{~d} r \\
= & \frac{\pi}{4} C s^{2} \delta^{4}
\end{aligned}
$$


To evaluate the micromodulus parameter $c$ in the equation above, we match, for the same homogeneous deformation, the peridynamic strain energy density with the classical strain energy density, $W=9 k s^{2} / 2$, where $k$ is a bulk modulus. The value for the $3 \mathrm{D}$ constant micromodulus is then derived as:

$$
c=\frac{18 k}{\pi \delta^{4}}
$$

In the bond-based peridynamics used in this paper, the particles interact only through a pair-potential. This assumption results in an effective Poisson ratio of $1 / 4$ in 3D, for an isotropic and linear micro-elastic material. This limitation can be removed as shown in [4], and it does not exist in the state-based formulation of peridynamics [11]. In peridynamics, material points are connected via elastic (linear or nonlinear) bonds and each bond can have a critical relative elongation, $s_{0}$ for modeling damage $([4,8])$. A bond breaks and no longer sustains force when its deformation is beyond this predefined limit $s_{0}$. Once a microelastic bond breaks, it stays broken. Because of this damage model, the deformation of a micro-elastic material is history-dependent (see [12]). The critical relative elongation parameter can be obtained by matching the peridynamic fracture energy (required to completely separate a body into two halves across a fracture plane by breaking all the bonds that initially connected points in the opposite halves, see [8]) with the measured fracture energy. The energy per unit fracture area in $3 \mathrm{D}$ for the complete separation of the body into two halves is the fracture energy, $G_{0}$. In 3D, Silling and Askari [8] relate $s_{0}$ with this measurable quantity, $G_{0}$.

$$
G_{0}=\int_{0}^{\delta} \int_{0}^{2 \pi} \int_{z}^{\delta} \int_{0}^{\cos ^{-1}(z / r)} \frac{C s_{0}^{2} r}{2} r^{2} \sin \varphi d \varphi d r d \theta d z
$$

For the constant micromodulus given in Equation (8), we obtain:

$$
s_{0}=\sqrt{\frac{5 G_{0}}{9 k \delta}}
$$

The critical elongation for a conical micro-modulus function in 2D is given in [9]. Observe that when some of the bonds at a node have been broken, the match with the material's fracture energy involves an integral over a smaller domain (the domain of nodes whose bonds with the central node have been broken are taken out). As a result, the critical relative elongation value would increase compared to the $s_{0}$ value obtained at a un-damaged node in the bulk. A damage-dependent critical relative elongation has been used in [13] where the influence on dynamic crack branching has been analyzed. The same damage-dependent model is used in the present study.

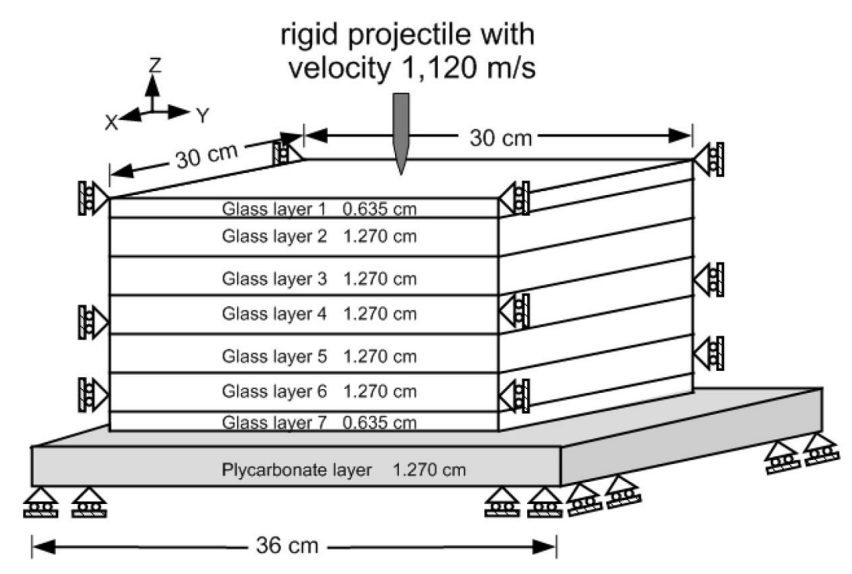

Figure 2. The geometry of the multi-layer glass target and boundary conditions used in the peridynamic model.

\section{Computational model and results}

\subsection{Model description}

A simplified three-dimensional model of the multi-layered glass system is created using the geometry and dimensions used in [1] and shown in Figure 2. While the geometry of the seven-layer glass system with a polycarbonate backing is the same as in the experiments, the boundary conditions are slightly different from experiments. For example, in the experiments the impact face of the top layer is taped so that the fragments are not lost upon impact. The membrane effect induced by the taping certainly influences the mechanical response, but whether this influence is of first or second-order remains to be seen. We do not impose conditions that mimic the taping of the front face. In addition, in the experiments the sides of the multi-layered system are also taped. We, instead, enforce zero in-plane displacements for nodes on this boundary. These conditions are more "rigid" than the experimental conditions and we expect more wave reflection from the boundaries than in the real test.

Moreover, in our simulations the polycarbonate layer has zero-vertical displacements imposed over where the polycarbonate extends beyond the glass (in the form of a square frame, see Figure 2), while in the experiments the polycarbonate is supported by wood blocks over the same region. Again, the experimental boundary conditions are more "absorbing" than our zero-displacements conditions. Note also that the boundary conditions used in [14] enforced zero-vertical displacements everywhere on the bottom of the polycarbonate layer.

In the experimental sample, the glass layers are bonded together with thin polyurethane layers $(0.6 \mathrm{~mm}$ thick $)$ which are not damaged, except for that between the first and second layers punctured by the projectile. Our cur- 
rent computational model does not include these thin layers, but modeling their presence with special types of peridynamic bonds is possible and will be attempted in the future. We, instead, use simple non-penetrating contact conditions at the interfaces between the glass layers with no bonding. Because of these slight differences in the boundary conditions and the structure of the glass laminate (the absence of taping and binding between the layers) we expect damage patterns to differ to a certain extent from the results reported in the experiments.

The simplest peridynamic linear-elastic with damage model is correlated to material properties for plate glass. The material properties for the glass material are: $\rho=2440 \mathrm{~kg} / \mathrm{m}^{3}$, Young's modulus $E=72 \mathrm{GPa}$, and energy release rate $G_{0}=15.47 \mathrm{~J} / \mathrm{m}^{2}$. Note that the results in Reference [14] used slightly different boundary conditions and a significantly higher fracture energy, one that corresponds to the fracture energy for soda-lime glass at crack branching (see [15]). A discussion comparing the present results with those in [14] is given in section 3.3 below. The bond-based peridynamic model used in this work leads to a fixed Poisson ratio of 0.25 (in 3D), which is near that of the soda-lime glass of 0.22 . For the polycarbonate (PC) layer we use a density of $1200 \mathrm{~kg} / \mathrm{m}^{3}$ and Young's modulus of $2 \mathrm{GPa}$. Since we use the bond-based version of peridynamics, the Poisson ratio of the PC backing material, which is about 0.37 , is replaced with the fixed value 0.25 . This will slightly affect the stress wave speed in the layer, but, as we shall see, no damage initiates in this layer and thus we believe the consequence of this approximation are secondary and the qualitative picture of damage progression in the multi-layered glass structure is not significantly affected.

A standard $12.7 \mathrm{~mm}, 13.4 \mathrm{~g}$, projectile with an $18 \mathrm{~mm}$ long cylindrical body is used in the experiments with an initial velocity of $1,120 \mathrm{~m} / \mathrm{s}$ ([1]). In our simplified numerical model we use a rigid projectile of the same dimensions (ogive tip). Note that in the experiments, the projectile deforms from its $18 \mathrm{~mm}$ initial length to $8 \mathrm{~mm}$ after being ejected from the target. The problem with a deformable projectile is left for the future.

The code EMU from Sandia, with some modifications described below, is used in all the peridynamic computational results shown in this work. For the sample with the geometry described above, we use a peridynamic horizon size of about $2.73 \mathrm{~mm}$, and a discretization size $\Delta x$ of about $0.91 \mathrm{~mm}$, giving a ratio $m=\delta / \Delta x=3.01$ and a total number of degrees of freedom of around 35 million ( 11.7 million nodes). We use a time step of about 0.07 $\mu$ s which ensures numerical stability for the forward Euler time-integration used in this work. For the numerical spatial integration of the peridynamic equations of motion Equation (1), we use the mid-point integration with a modified approximation of the nodal volume as proposed in [16]. For the damage model we use the strengthening of the bonds in damaged regions as discussed in Equation (11) from [13].

The nonlocal region size, the horizon, is probably large for this type of material in order to reproduce the crack propagation speeds from the real test. Note that the authors of [1] only discuss the damage morphology in the glass layers after the impact; the propagation speed of the failure fronts or of individual cracks is not monitored. The reason for the dependence of the propagation speed on the horizon size is discussed in [5], where it is argued that when dynamic crack growth in brittle materials is induced by stress waves interacting with the fracture, then, due to trailing waves behind the stress wave front (which depend on the size of the nonlocal region and break bonds), the crack propagation speed is higher the larger the horizon is (see [9] and [13]). When, however, the crack propagation is not influenced by stress waves (as in the case when loads are applied on the crack faces and the propagation is monitored until the stress waves return from the boundaries of the structure to meet the crack tip), then the crack propagation speed is independent of the horizon size ([5]). A horizon size that produces sufficiently small dispersion (in the form of trailing waves) that these trailing waves do not interfere much with the crack propagation speed, is in the range of $0.5-0.1 \mathrm{~mm}([9])$ for glasses. With a discretization size at least $1 / 3$ or $1 / 4$ of the horizon size, the resulting computational model would be two orders of magnitude larger than the ones used here. The available computational resources at this time $(322.2 \mathrm{GHz} \mathrm{Op}-$ teron processors) did not allow us to use such a horizon size and such a discretization. We note, however, that the damage map is less sensitive to the horizon size ([14]) than the propagation speed, and this is what we report on here and compare with the experiments performed in [1].

It is important to note that we do not use any explicit material strain-rate dependency in this model. Any strainrate dependence in the results will be due to the intrinsic dynamics of wave propagation, damage, and fracture in the brittle layered system. In a sense, the results shown below will serve as a test of the idea that, at high strainrates, glass is effectively behaving as a quasi-brittle material and that no explicit material strain-rate dependence is needed in modeling the mechanical behavior under such loading conditions.

\subsection{A comparison between FEM and peridynamics for the elastic response of a multilayered system to impact}

Before going into the damage problem, we analyze whether the nonlocal solution for the problem of elas- 
tic impact, in which no damage is allowed to initiate, with the horizon size specified above, leads to an elastic bending deformation field similar to one obtained from a dynamic (explicit) Finite Element Analysis (FEM) using Abaqus. For this purpose, we monitor the bending deformation in the problem of elastic impact of a spherical rigid projectile onto a multi-layered plate system similar to the one described above. In both the FEM and the peridynamic models, we exclude the possibility of damage. Using the horizon size and grid spacing mentioned in the previous section, which will be used for the seven-layer structure as well, we consider a "reduced" system comprised of only two layers of glass $(30 \mathrm{~cm}$ by $30 \mathrm{~cm}$ by $1.27 \mathrm{~cm}$ ) and the polycarbonate backing layer $(36 \mathrm{~cm}$ by $36 \mathrm{~cm}$ by $1.27 \mathrm{~cm}$ ). The same boundary conditions as in the seven-layer system are used (see Figure 2 ), and linear elastic and linear micro-elastic constitutive models are used for both the FEM and the Peridynamic solutions.

For the FEM solution, we perform a convergence study with quadratic elements in which the mesh size (in the $x-y$-plane) varies from $1.8 \mathrm{~cm}$ (coarse), to $0.9 \mathrm{~cm}$ (medium-coarse) to $0.45 \mathrm{~cm}$ (medium-fine) to $0.15 \mathrm{~cm}$ (fine). The first two meshes use only one element through the thickness of each glass layer and PC backing, while the medium-fine and fine meshes have three and eight elements through the thickness of each layer, respectively.

A rigid sphere of $2.5 \mathrm{~cm}$ radius and the mass density of steel, is impacting the center of the system at $100 \mathrm{~m} / \mathrm{s}$. The Figure 3 shows the vertical displacement of the centroid of the first glass layer, in time, obtained by the several FEM solutions from Abaqus (with finer and finer meshes) and from the peridynamic model that uses the same horizon and grid spacing as those used for the seven-layer structure below. We observe that the peridynamic solution with this horizon is nearly identical with the converged FEM solution in the initial stages of the deformation caused by the impact. Some differences, of about $10 \%$ appear at the later times possibly due to the different contact algorithms used in EMU and Abaqus. It is important to note, however, that at the deformation levels obtained after a few microseconds or tens of microseconds from the impact, glass is starting to fracture. Therefore, our attention in this verification test of the elastic behavior, only needs to focus on the first few tens of microseconds from the impact. During this interval, the match between the converged FEM solution and the Peridynamic result is very good. We conclude that the peridynamic horizon and the discretization mentioned in the previous section and to be used in modeling the impact on the seven-layer below, are sufficient to capture the correct elastic bending deformation of a multi-layer structure, in the absence of damage. The comparison between the projectile speed is also shown in Figure 3.
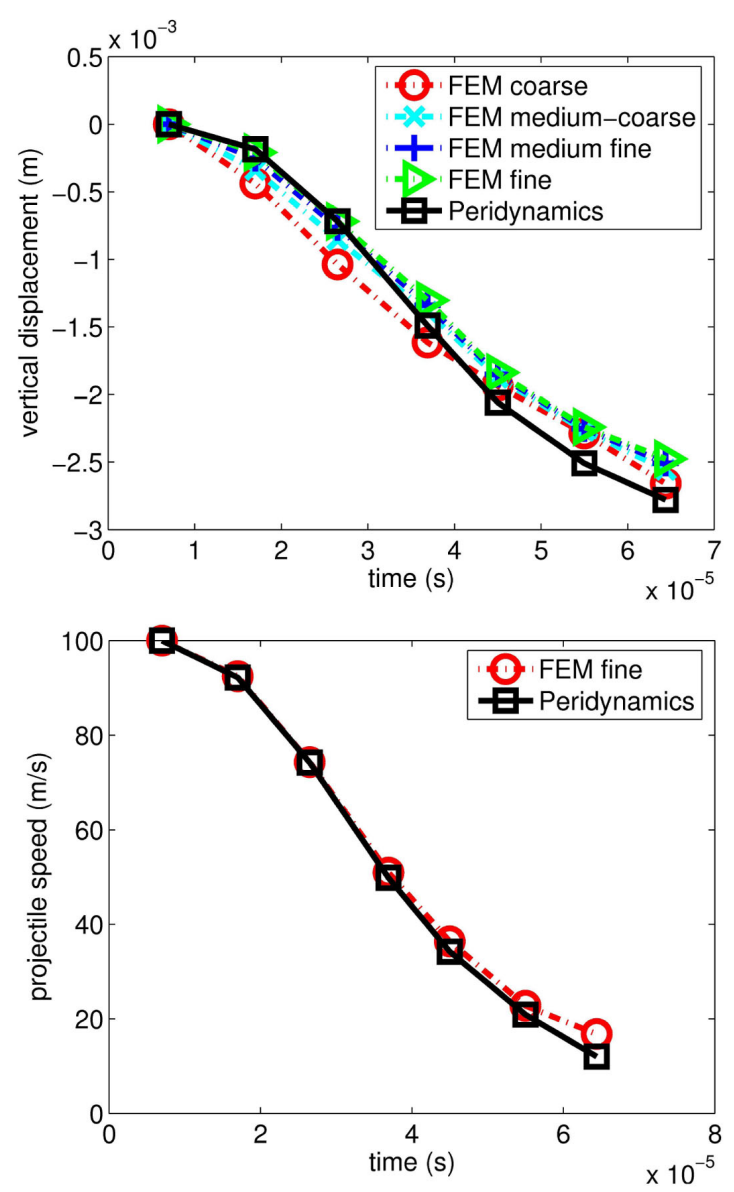

Figure 3. Elastic impact of a rigid sphere onto a two-layer glass with a PC backing system. Top: comparison of the vertical displacement for the centroid of the first glass layer between the finite element method and peridynamics. Bottom: projectile velocity evolution.

\subsection{Computational results for the damage progres- sion in the seven-layer system}

The projectile impact generates strong stress waves that propagate in the seven-layer glass laminate interact with, and reflect from, the boundaries, the interfaces between the layers, and the newly created crack surfaces.

We now present and examine the peridynamic damage evolution results for this problem in terms of the damage index. The peridynamic damage index is computed at every discretization node and at every time-step, as the number of broken peridynamic bonds divided by the total number of original bonds for that particular node. This definition means that the damage index is between zero and one. The legend shown in Figure 4 is used in all damage plots. A damage index of about 0.5 at points aligned along a certain direction means that a crack separating two surfaces has been created. If, instead, the damage index map shows no localization in a certain area, that means that a diffuse-type damage has occurred there. 


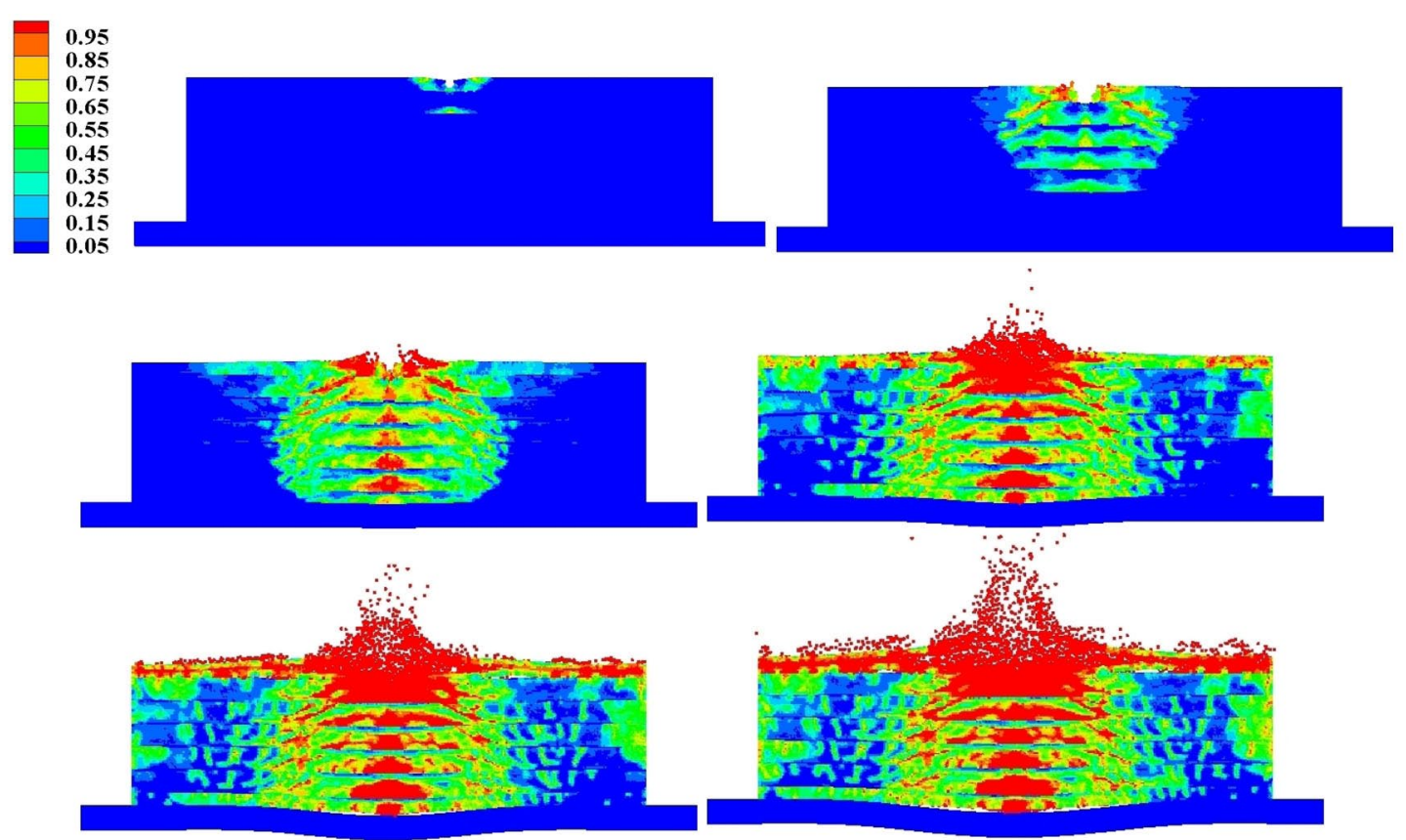

Figure 4. The time-evolution of the damage map for the through-thickness cross-section (displacements are amplified in this figure by a factor of 2 for better visualization). From left to right, snapshots taken at $12,27,43,79,110$, and $161 \mu$ s from the contact between the projectile and the glass laminate. The legend for the damage map used here is used in all remaining figures. See movie 1.

We compare our results for the simplified model discussed before of the sample used in the experiments of [1] with those discussed in [1], where a detailed post-mortem analysis of the damage that has occurred after impact is provided. In contrast with the experiments, the simulation results allow us to observe not only the final damaged stage, but also how damage and fracture evolve in time. This is an important advantage of simulations results since accurate modeling of dynamic behavior under such extreme loading conditions can lead to better designs for multi-layered brittle systems.

\subsubsection{Damage evolution for the cross-section}

A cross-sectional cut plane $(x-z$ or $y-z$ plane in Figure 2) through the middle of the sample is used to plot the damage map index through the glass layers and the polycarbonate backing in Figure 4 as it progresses in time. An interesting feature is revealed: the damage progresses through the thickness of each layer from the bottom up, indicating an important role played by the interfaces (and wave reflections from them) and by the bending deformation, which helps in propagating the cracks from the bottom surface to the top face of each layer. We observe the formation of a Hertz-type conical crack similar to what is observed in the experiments (Figure 3 in [1]). The projectile is ejected from the sample at around $23 \mu \mathrm{s}$, but most of the damage takes place after this event. In experiments, the projectile stops in the second layer after penetrating about $19 \mathrm{~mm}$ and is ejected, while in our peridynamic simulations the projectile stops in the second layer after penetrating a depth of about $17 \mathrm{~mm}$, and is ejected.

Notice that no damage initiates in the polycarbonate layer, similar to what is observed in the experiments. The bending deformation is clearly visible and it is interesting to compare these results with those in [14] where, besides the tougher glass used, the bottom of the polycarbonate layer is constrained from moving in the z-direction. Due to limitations of the computational resources we only monitor the damage progression for the first about 200 $\mu \mathrm{s}$ from the moment of impact. The elastic "snap-back" of the polycarbonate layer that follows after that, will likely lead to further evolution of damage but this will to be confined to enlarging the already formed cracks since most of the energy has already been consumed in creating the damage observed until the end-time of our simulation. Note that the projectile is not ejected by the rebound of the PC backing plate, which happens much later, but by the rebound of the first few glass layers in the first few tens of microseconds.

In our model we do not tape the top face nor do we tape the sides of the glass layers together (instead we use zero-in-plane displacements on the sides), therefore fragments from the first layer are flying away. Note, however, that the first layer does not bounce away from the 

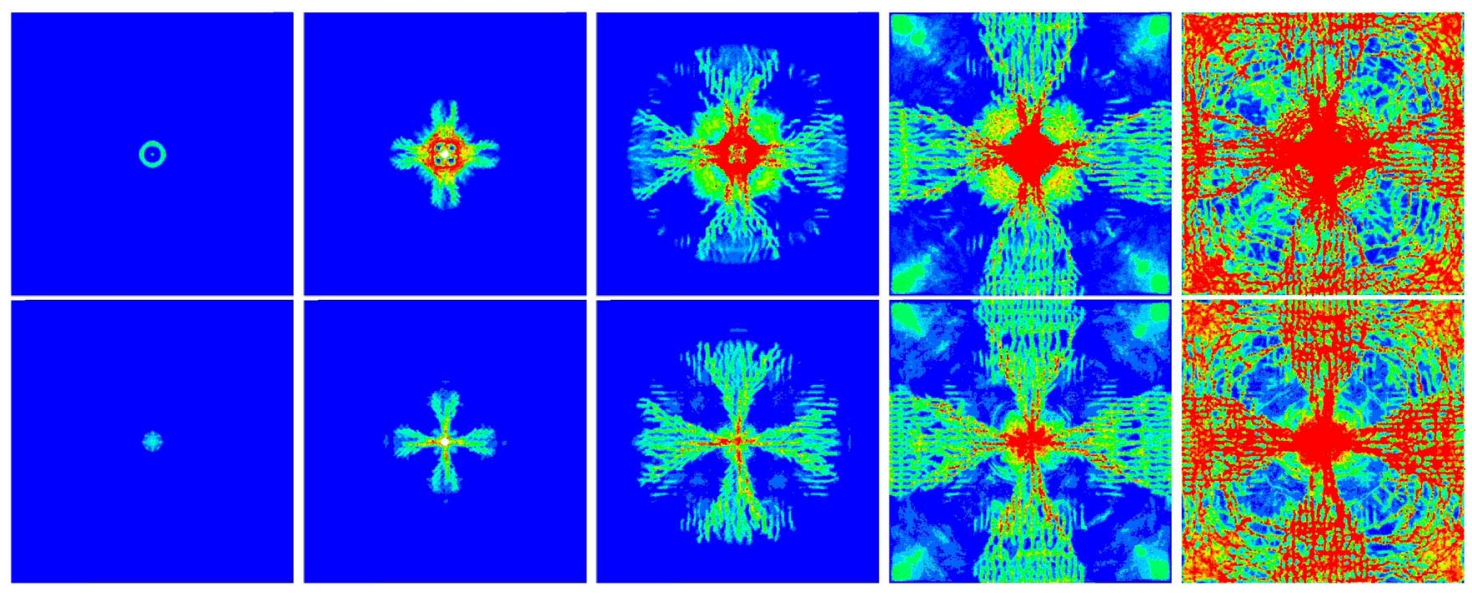

Figure 5. The time-evolution of the damage map for the top face (top row) and bottom face (bottom row) of the first layer. From left to right, snapshots taken at $9,27,43,60$, and $127 \mu$ s from the contact between the projectile and the glass laminate. See movie 2 for the top face.

rest of the structure as in the case in which the polycarbonate layer is constrained over its entire bottom face, as is done in [14]. Spall-type fractures in the cross-section of the first layer are observed. These are cracks parallel to the surface of the layer. The absence of the taping on the impact face in our model likely contributes to the formation of these cracks. In the experiments, cracks parallel to the surface are seen in the deeper layers, including he last layer. We attribute the difference between the computations and the experiments to the absence of bonding between the layers in our simplified peridynamic model compared to the polyurethane bonding used in the experiments. The experiments indicate that surface chips are ejected from some of the cracks in the front plate, which the authors of [1] attribute to the late-time bending from the rebound that is also responsible for the ejection of the projectile. They also attribute transverse cracks to the rebound bending. The cross-sectional views in Figure 4 show that some of these fragments from the top layer, and some of the transverse cracks (see more about transverse cracks below) form in the initial bending. The rebound bending certainly adds to these types of failure. We also note that since we do not bond the layers together, as is done in the experiments, the interdependence of the rebound between the layers is weaker in our simulations than it is in the experiments. In fact, the rebound of the PC backing layer does not even take place in the first 200 $\mu$ s after the impact.

The cause for the asymmetry seen in the computational damage map in Figure 4 and all subsequent figures has been discussed in [13].

\subsubsection{Damage evolution in the first layer}

It is interesting to observe the evolution of damage in each individual layer. For the first layer the evolution of damage is shown in Figure 5 We observe that damage evolves differently between the top face and the bottom face of the first glass layer, and, as we shall see, this is happening in every glass layer. In this first layer, radial cracks start on the bottom face, they continue on both faces as bundled cracks that start to fan out as they propagate towards the boundaries of the layer. This is very similar to the schematic description from the experiments, shown in Figure 7 in [1].

Notice also that the "floret"-type crack on the bottom face is the same as that observed in [1] on the bottom of the last layer. As we shall see, the failure mode at the bottom of each layer starts as an asterisk. We conclude that this type of crack is a result of the stress waves that propagate through the thickness and interact with the surface of the layer. Of course, the floret crack in the first layer is subsequently "erased" by the progression of the perforation through the first and part of the second layer, while in the other layers it is engulfed by further damage. Note that the cracks that have a similar location on the top and bottom faces of a glass layer are straight through-thickness cracks. If, on the other hand, the trace of damage on the top and bottom surface of a layer are similar but not perfectly aligned, then the conclusion is that these cracks are tilted in the thickness direction.

Some notable differences are also seen with the results in [14] that were obtained with a much higher fracture energy for the glass (at branching) and zero vertical displacement on the entire bottom face of the PC backing layer. In the tougher glass case, there are significantly fewer "dicing cracks" than we observe now, and much less damage in general. The different boundary conditions below the polycarbonate layer used in [14] led to a rebound of the first layer which is visibly pushed away from the rest of the structure. 

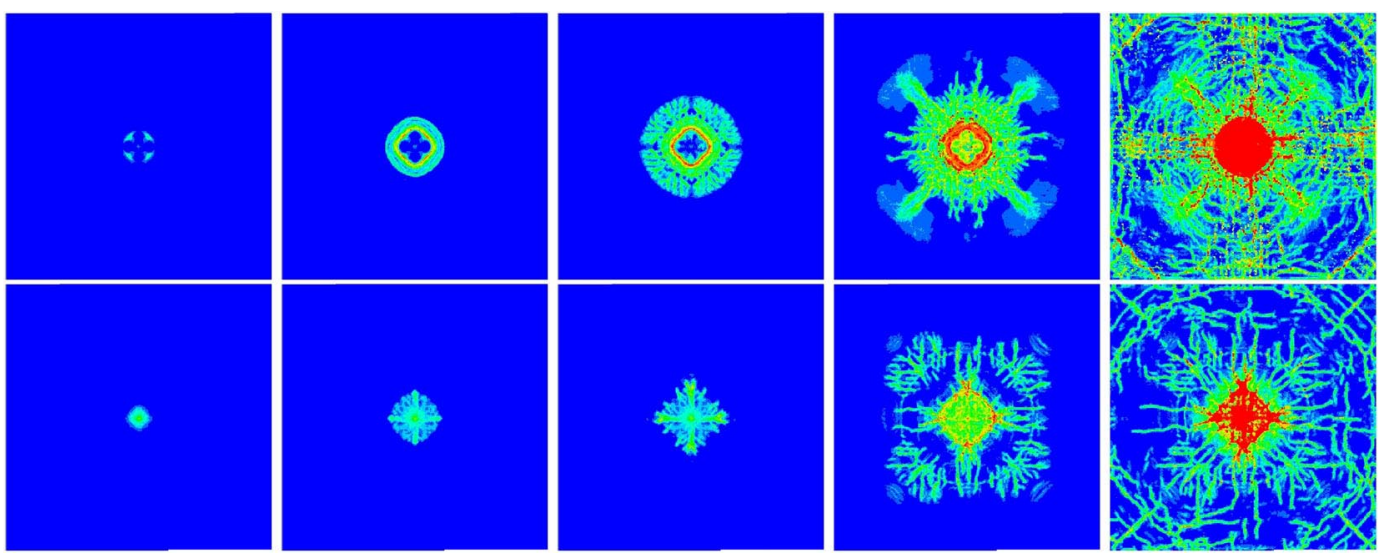

Figure 6. The time-evolution of the damage map for the top face (top row) and bottom face (bottom row) of the second layer. From left to right, snapshots taken at $12,18,24,43$, and $127 \mu$ s from the contact between the projectile and the glass laminate. See movie 3 for the top face.

\subsubsection{Damage evolution in the second layer}

The second layer is experiencing very different types of damage compared with the first layer. The bundled radial cracks that fan out are not present in this layer; instead, a wider central region experiences a more diffusetype damage (see Figure 6). This type of damage may be indicative of the needle-type cracks observed in the experiments in this region only. These cracks create fragments with dimensions of $1 \times 1 \times 20 \mathrm{~mm}$ that cannot be captured by the current computational resolution of our model. In this second layer the projectile stops and is ejected from the target. We mention that damage continues to evolve even after the $127 \mu$ s mark shown last in Figure 6, but its structure remains basically unchanged. At around $200 \mu$ s all damage activity quiets down.

Away from central diffuse-damage zone a region with coarser radial cracks forms, and even closer to the edges, dicing cracks become prevalent. This damage morphology closely resembles that described in the experiments in [1]. Some of the cracks grow from the boundaries of the structure, and especially easy to identify are the cracks in the corners, at $45^{\circ}$ angles. These cracks are consistent with wave reflections from the corners and they are also seen in Figure 10 from [1] which is a picture of the third layer. The corners of the second layer are not shown in [1].

\subsubsection{Damage evolution in the fourth layer}

For the inner layers, 3rd to 6th, we select the 4th as a representative layer. Indeed, the computational results show that much similarity between the damage patterns in these layers exists, and that has also been observed in the experiments [1]. Circumferential cracks (called ripple cracks in [1]) are highly prominent in these inner layers and they surround a central region of initially undamaged glass on the top face, as reported in the experiments. The dicing cracks reported in the experiments (see Figure 10 in [1]) are also obtained by the peridynamic simulations near the boundaries of the layer. These cracks have the same signature on the top and bottom faces of the layer, therefore they are vertical through-thickness cracks.

The central region, with a diameter similar to the crater in the second layer, on the top surface of this layer and all the layers from third to seventh shows significantly less damage than the corresponding region on the bottom face of the layer (see top row of plots from Figure 7 and Figure 8). These features correspond to the "compact disks" found in the experiments of Bless and Chen [1]. The lower parts of these regions are heavily damaged. The cause for these features is the wave propagation and reflection across the interfaces.

Something that is not captured by our simulation are the massive quadrant cracks (radial cracks along the horizontal and vertical directions) seen in Figure 11 in [1]. One explanation for this is the absence of bonding between the layers in our simplified computational model. The bonding used in the experiments would lead to a more coordinated bending motion between the layers, thus creating the conditions for quadrant cracks to be produced after the rebound of the entire system. As observed from Figure 4, by the end of our simulation the backing PC layer has not yet rebounded, while the top layers have already done so. Continuing the simulation until the PC layer rebounds may still induce some further quadrant cracking, but in the absence of bonding the more likely scenario is the layers being pushed apart from each other. In the future we plan to include special peridynamic bonds between the layers to mimic the presence of the thin polyurethane layers used in the experiments. We also note that quadrant crack appear in the seventh layer (see below) but they are not massive crevices, and stop short of reaching the boundaries, at least by the end of our simulation. 

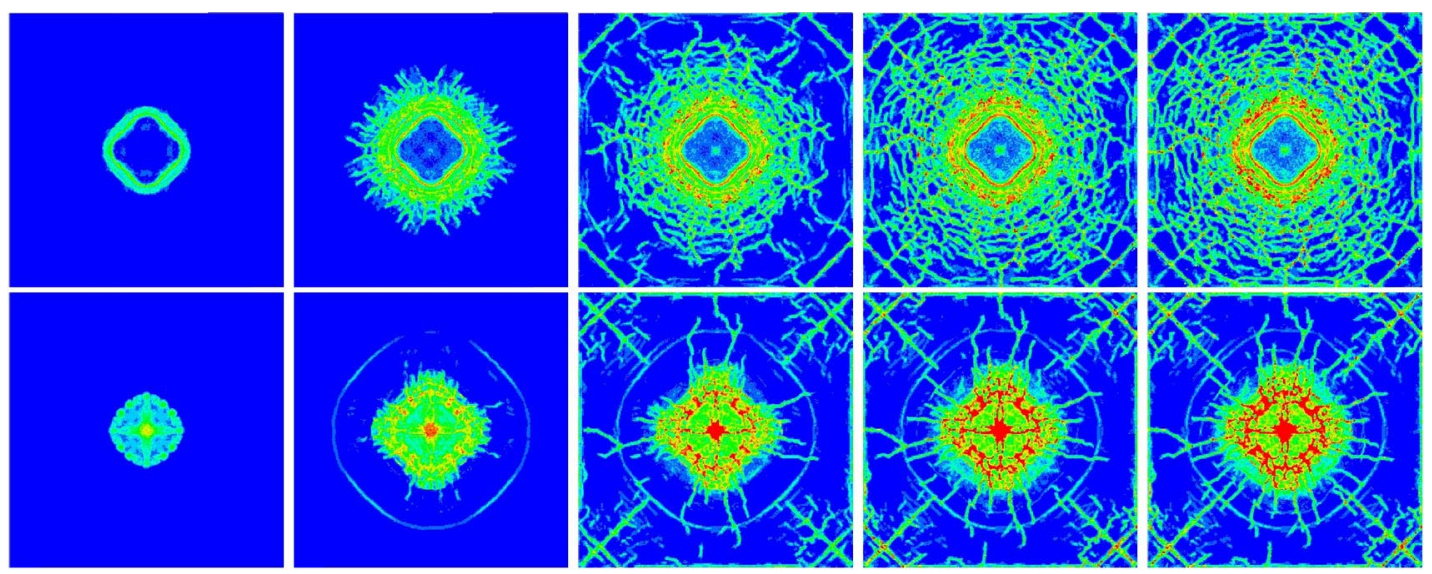

Figure 7. The time-evolution of the damage map for the top face (top row) and bottom face (bottom row) of the fourth layer. From left to right, snapshots taken at $27,43,79,127$, and $161 \mu$ s from the contact between the projectile and the glass laminate. See movie 4 for the top face.
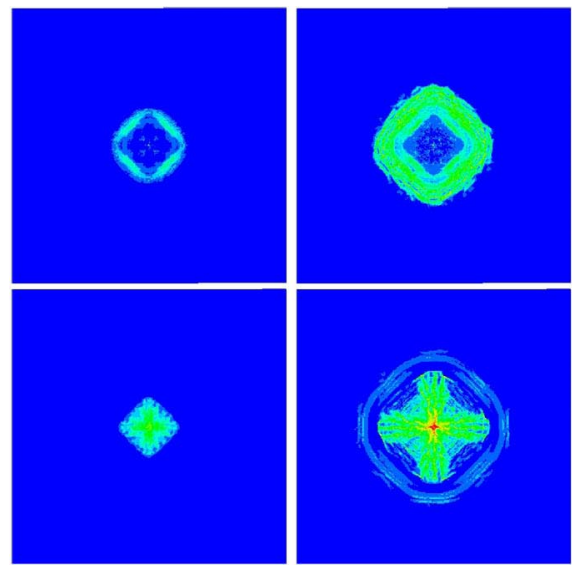
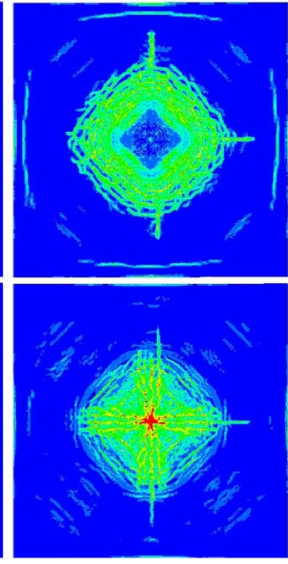
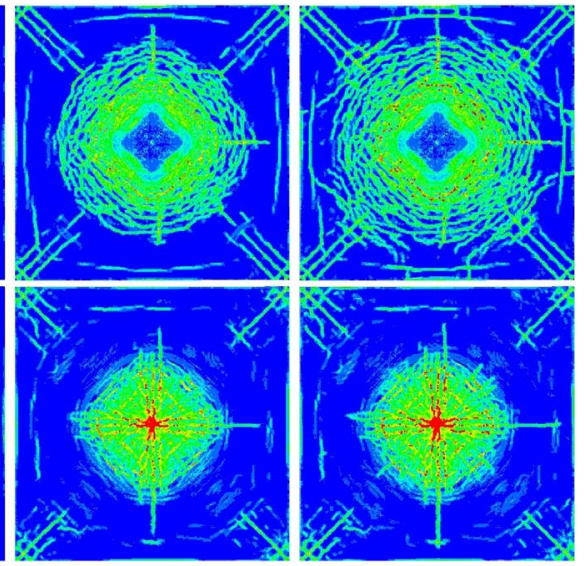

Figure 8. The time-evolution of the damage map for the top face (top row) and bottom face (bottom row) of the last glass layer (7th). From left to right, snapshots taken at $36,50,79,110$, and $161 \mu$ s from the contact between the projectile and the glass laminate. See movie 5 for the top face.

\subsubsection{Damage evolution in the seventh layer}

In the last layer of the multi-layered glass systems, which has the same thickness as the first one and half the thickness of the other layers, we observe the generation of a number of very straight radial cracks, along the quadrant cracks directions seen in the experiments (see Figure 8). Bless and Chen [1] noted: "unlike the strike plate, radial cracks on the last plate were very straight, were continuous, and extended all the way to the edges". Unlike the experiments, however, our results show such radial cracks running to the edges only for the bisector directions (those ending at the corners) and, to a lesser extent, the cracks along the $x$ and $y$ directions. This difference from the experiments is likely due to the absence of bonding between the layers in our simplified computational model and also due to the taping of the lateral sides of the system used in the experiments. The order in which these cracks form in the simulations is also interesting: the straight cracks running to the corners appear last, and, in fact, they grow from the corners towards the center! Especially on the top face, parallel cracks with spacing of about $1 \mathrm{~cm}$ are observed to develop in the late stages. Similar spacing between continuous radial cracks are reported in [1] in bands that bisect the edges into quadrants.

In the center of the bottom face of this last layer, an asterisk-like feature is seen to develop. Such features are reported in [1] where it is noted that these do not appear to be through-thickness cracks. The same is seen from our results in Figure 8. Dicing cracks develop in this layer as well and they tend to be more prevalent near the edges of the layer. 
Compared to the results obtained with a much tougher glass (see [14]), here the results indicate an amount and spread of damage comparable with what is observed from Figure 8 in the experimental paper [1].

\section{Conclusions}

High velocity impact on multi-layer glass systems produces a variety of damage types with distinct patterns in every layer of the glass laminate. We use a simplified peridynamic model of a multi-layered glass structure for which experiments have been reported recently in the literature. We first test the peridynamic model against a dynamic Finite Element solution for the elastic impact on a multi-layered system. We then use brittle damage in the peridynamic model and compare the results with the damage morphologies in the seven-layer glass system with a polycarbonate layer backing observed in the experiments. Layer-by-layer, most of the fracture patterns observed in experiments are well reproduced by the simplified peridynamic model. The peridynamic simulations shows that the dominant mechanisms that induce and influence the damage evolution in the impact problem on a multi-layered brittle structure are: (a) the dynamics of stress waves and their interactions with the interfaces between layers, the boundaries, and the newly created crack surfaces, and (b) the structural response (flexural waves) of the glass layers and polycarbonate backing plate. The brittle damage progression is sensitive to the boundary conditions.

Compared to the experimental conditions, the simplified model does not include the taping of the strike face of the first layer, and it replaces the taping of the sides of the layers with zero in-plane displacements. Moreover, in the computational model the layers are not bonded by the polyurethane layers used in the experiments and, because of that, the rebound behavior of the glass layers and the polycarbonate backing plate is different than in the experiments. These differences, however, do not preclude the simplified peridynamic model to capture most of the essential damage characteristics that take place from the high-velocity impact on the multi-layered glass system. Some finer features of damage observed in experiments, such as bow-tie cracks and needle cracks, are not captured, due to the insufficient resolution in the numerical model.

It is important to note that the results obtained here do not use any explicit material strain-rate dependencies. All the strain-rate dependence in the results is due only to the implicit dynamics of wave propagation, damage and fracture in the brittle multilayer system. The closeness between the peridynamic results presented here and the experimental results can be also interpreted as a validation of the point that, at the strain rates induced by high-velocity impact, glass behaves very closely as a quasi-brittle material and that no explicit material strain-rate dependence is needed in modeling the mechanical behavior under such loading conditions.

The peridynamic results presented here demonstrate that it is becoming possible to obtain predictive simulations of dynamic fracture and damage in multi-layered brittle materials from high-velocity impact. Future plans include modeling the bonding between the glass plates, which has an important influence on the bending and rebound of the structure and on stress wave dissipation, which influence the damage evolution in the multi-layered system, and using a deformable projectile instead of a rigid one.

Acknowledgments - The authors are thankful for the financial support offered through research contracts between UNL and the ARO (Dr. Larry Russell), and ARL (project coordinators Dr. C.F. Yen and Dr. C. Randow), ARO award number 58450EG. The work of Y. D. Ha was also supported by research funds from Kunsan National University, Korea. The computations in this work were completed utilizing the Holland Computing Center of the University of Nebraska. The authors also thank the three anonymous reviewers for Central European Journal of Engineering whose comments and suggestions led to an improved manuscript.

\section{References}

[1] Bless S., Chen T., Impact damage in layered glass, Int. J. Fract., 2010,162(1), 151-158

[2] Patel P. J., Gilde G. A., Dehmer P. G., McCauley, J. W., Transparent ceramics for armor and EM window applications, In: Proceedings of SPIE, 2000, 4102

[3] Grinfeld M.A., McCauley J. W., Schoenfeld S. I., Wright T. W., Failure patterns in brittle ceramics and glasses. In: Proceedings of the 23rd international symposium on ballistics, Tarragona, April 2007

[4] Silling SA, Reformulation of elasticity theory for discontinuities and long-range forces, J. Mech. Phys. Solids, 2000, 48(1), 175-209

[5] Bobaru F., Hu W., The meaning, selection, and use of the peridynamic horizon and its relation to crack branching in brittle materials, Int. J. Fract., 2012, 176, 215-222

[6] Silling S. A., Lehoucq R. B., Peridynamic theory of solid mechanics, Adv. Appl. Mech., 2010, 44, 73-168.

[7] Silling S. A., Zimmermann M., Abeyaratne R., Deformation of a peridynamic bar, J. Elast., 2003, 73(1), 173-190 
[8] Silling S. A., Askari, E., A mesh-free method based on the peridynamic model of solid mechanics, Comput. Struct., 2005, 83(17-18), 1526-1535

[9] Ha Y. D., Bobaru, F., Studies of dynamic crack propagation and crack branching with peridynamics, Int. J. Fract., 2010, 162(1), 229-244

[10] Bobaru F., Yang M., Alves L. F., Silling S. A., et al., Convergence, adaptive refinement, and scaling in 1d peridynamics, Int. J. Numer. Meth. Engng., 2009, 77(6), 852-877

[11] Silling S. A., Epton M., Weckner O., Xu J., Askari E., Peridynamic states and constitutive modeling. J. Elast., 2007 88(2), 151-184

[12] Silling S. A., Bobaru F., Peridynamic modeling of membranes and fibers. Int. J. Non-Linear Mech., 2005, 40, 395-409.
[13] Ha Y. D., Bobaru F., Characteristics of dynamic brittle fracture captured with peridynamics, Eng. Fract. Mech., 2011, 78, 1156-1168.

[14] Bobaru F., Ha Y. D., Hu W., Peridynamic modeling of dynamic brittle fracture in a multi-layered glass system, In: Proceedings of SAMPE (May 21-24, 2012, Baltimore USA), 2012.

[15] Bowden F. P., Brunton J. H., Field J. E., Heyes A.D., Controlled fracture of brittle solids and interruption of electrical current, Nature, 1967, 216, 38-42.

[16] Bobaru F., Ha Y. D., Adaptive refinement and multiscale modeling in $2 \mathrm{~d}$ peridynamics, Int. J. Multiscale Comp. Eng., 2011, 9(6), 635-659. 